\title{
Unexpected hemorrhage during robot-assisted laparoscopic prostatectomy: a case report
}

\author{
Shoko Nakano, Junko Nakahira*, Toshiyuki Sawai, Noriko Kadono and Toshiaki Minami
}

\begin{abstract}
Background: Robot-assisted laparoscopic prostatectomy is increasingly performed as a minimally invasive option for patients with organ-confined prostate cancer. This technique offers several advantages over other surgical methods. However, concerns have been raised over the effects of the steep head-down tilt necessary during the procedure. We present a case in which head-down positioning and abdominal insufflation masked the signs of an intraoperative hemorrhage.
\end{abstract}

Case presentation: A 73-year-old Asian man developed severe hypotension caused by an unexpected hemorrhage during robot-assisted laparoscopic prostatectomy for prostate cancer. Although our patient's blood pressure steadily decreased during the procedure, his systolic blood pressure remained above $80 \mathrm{mmHg}$ while he was tilted head downward at an angle of $28^{\circ}$. However, his blood pressure dropped immediately after he was returned to the horizontal position and abdominal insufflation - to create a pneumoperitoneum - was ceased at the end of surgery. We returned the patient to a head-down tilt to keep his blood pressure stable and began fluid infusion. Blood test results indicated that a hemorrhage was the cause of his hypotension. Open abdominal surgery was performed to stop the bleeding. The surgeons found blood pooling inside his abdomen from a longitudinal cut in a small arterial vessel in his abdominal wall, possibly a branch of his external iliac artery. The surgeons successfully controlled the hemorrhage and our patient was moved to our intensive care unit. Our patient recovered completely over the next few days, without any neurological deficits.

Conclusions: We suspect that blood began to pool in our patient's superior abdomen during surgery, and that increased intra-abdominal pressure suppressed the hemorrhage. When our patient was returned to the horizontal position and insufflation of his abdomen was discontinued, the resulting increased rate of hemorrhage caused a sudden drop in blood pressure. Surgeons and anesthesiologists must understand the hemodynamic changes that result from head-down patient positioning and abdominal insufflation.

Keywords: Operative hemorrhage, Robot-assisted laparoscopic radical prostatectomy, Steep head-down tilt, Case report

Abbreviation: RALP, Robot-assisted laparoscopic prostatectomy

\footnotetext{
* Correspondence: ane052@osaka-med.ac.jp

Department of Anesthesiology, Osaka Medical College, 2-7 Daigaku-machi,

Takatsuki, Osaka 569-8686, Japan
} 


\section{Background}

Robot-assisted laparoscopic radical prostatectomy (RALP) is increasingly performed as a minimally invasive surgery option for organ-confined prostate cancer. RALP has various advantages over other surgical methods, including decreased blood loss and pain, shorter operation time and hospital stay, and lower complication rates [1]. However, concerns have been raised about the steep head-down tilt necessary during the procedure. This tilting can prevent recognition of deteriorating vital signs, causing complicated effects on patient hemodynamics, such as increased venous return or suppression of increased venous return during pneumoperitoneum. We report a case of delayed discovery of a hemorrhage caused by injury to a branch of the external iliac artery during RALP.

\section{Case presentation}

A 73-year-old, 152.8-cm, 56.5-kg Asian man with a history of aortic valve insufficiency, hypertension, and postoperative deep vein thrombosis was scheduled for RALP to treat prostate cancer. His prostate-specific antigen concentration was $4.1 \mathrm{ng} / \mathrm{mL}$. His prostate cancer had been diagnosed by biopsy 6 months earlier and his Gleason score was $4+3=7$. He was undergoing complete androgen blockade therapy. Preoperative laboratory results showed a hemoglobin concentration of $13.5 \mathrm{~g} / \mathrm{dL}$, hematocrit of $38.8 \%$, and no abnormalities in his hemostatic function.

On the day of surgery, anesthesia was induced intravenously with $100 \mathrm{mg}$ of propofol, $50 \mathrm{mg}$ of rocuronium, and a constant-rate infusion of remifentanil at $0.3 \mu \mathrm{g} / \mathrm{kg} /$ min with inhaled desflurane at $5 \%$. We maintained anesthesia with inhaled desflurane at $5 \%$ and intravenously administered remifentanil at $0.2 \mu \mathrm{g} / \mathrm{kg} / \mathrm{min}$ in a fraction of inspired oxygen of 0.45 . During surgery, our patient was positioned with a head-down tilt of $28^{\circ}$.

After almost 3 hours of surgery, at the surgical stage of urethrovesical anastomosis, our patient's systolic blood pressure was $<70 \mathrm{mmHg}$. At that moment, we administered $20 \mathrm{mg}$ ephedrine, $300 \mu \mathrm{g}$ phenylephrine, and $0.03 \mu \mathrm{g} / \mathrm{kg} / \mathrm{min}$ norepinephrine to maintain adequate blood pressure. Arterial blood gas measurements showed a hemoglobin concentration of $10.2 \mathrm{~g} / \mathrm{dL}$ and hematocrit of $30 \%$. We did not detect any problems with his respiratory parameters. Dissection of his pelvic lymph node was performed. The surgeons confirmed hemostasis.

The surgery was completed after $\sim 4$ hours, abdominal insufflation to create the pneumoperitoneum was ceased, and the operating table was returned to the horizontal position. Our patient's blood pressure immediately dropped, with his systolic blood pressure decreasing to $40 \mathrm{mmHg}$. Repeat blood tests showed a hemoglobin concentration of $7.2 \mathrm{~g} / \mathrm{dL}$ and hematocrit of $21 \%$. After administration of vasopressors, we placed a central venous catheter, with the patient in the head-down tilt position.
We administered $120 \mu \mathrm{g}$ of norepinephrine. After administration, our patient's hemoglobin level fell to $5.9 \mathrm{~g} / \mathrm{dL}$, his hematocrit fell to $16.9 \%$, and his platelet count was $56 \times 10^{3} / \mathrm{dL}$. Because these findings indicated a possible hemorrhage, the surgeons immediately initiated open surgery. A large volume of blood and blood clots were present in his abdominal cavity and so we began rapid transfusion. His systolic blood pressure remained at $40 \mathrm{mmHg}$ for $10 \mathrm{~min}$; we performed chest compressions to maintain his blood pressure. The surgeons reduced the bleeding and maintained his blood pressure by manually pinching his common iliac artery and external iliac artery on his right side. They discovered a longitudinal slice in a branch of his right external iliac artery along the internal oblique muscle; ligation of this vessel achieved hemostasis. The branch was located around an instrument port that was located medially between a camera port and laterally to an assistant port.

Surgery concluded after confirmation of hemostasis and blood pressure stability. The operation lasted 7 hours 50 minutes; anesthesia, 10 hours 14 minutes; and pneumoperitoneum, 3 hours 51 minutes. Our patient lost $5650 \mathrm{~mL}$ of blood and $350 \mathrm{~mL}$ of urine during surgery. Blood loss was estimated by measuring the blood in the suction bottle and gauze used to absorb the blood and blood clots. He received $5320 \mathrm{~mL}$ of red blood cells, $2400 \mathrm{~mL}$ of fresh frozen plasma, and $2500 \mathrm{~mL}$ of a $5 \%$ albumin solution intraoperatively. His blood pressure, heart rate, and percutaneous oxygen saturation were recorded in the anesthetic chart (Fig. 1).

After surgery, our patient was admitted to the intensive care unit. The following day he was extubated and returned to the general care ward. He recovered from surgery with a suspected ileus and was discharged on day 29.

\section{Discussion}

RALP is increasingly performed in patients with prostate cancer because it is a less invasive procedure than other prostatectomy methods. A previous study showed that RALP results in better surgical outcomes and fewer complications than laparoscopic radical retropubic prostatectomy [1]. Several postoperative complications, including pulmonary edema, central nervous system dysfunction caused by the steep head-down tilt $\left(25-40^{\circ}\right)$, and abdominal pneumoperitoneum with carbon dioxide, have been reported [2-5]. Anesthesiologists carefully avoid these complications during surgery and address postoperative complications promptly.

Many studies comparing RALP with retropubic radical prostatectomy have found that RALP reduces blood loss during surgery [3, 6-8]. Typical blood loss during RALP at our institution is less than $300 \mathrm{~mL}$, including that passed through the urine. Patients generally do not need a transfusion, as many studies have reported. Our case 


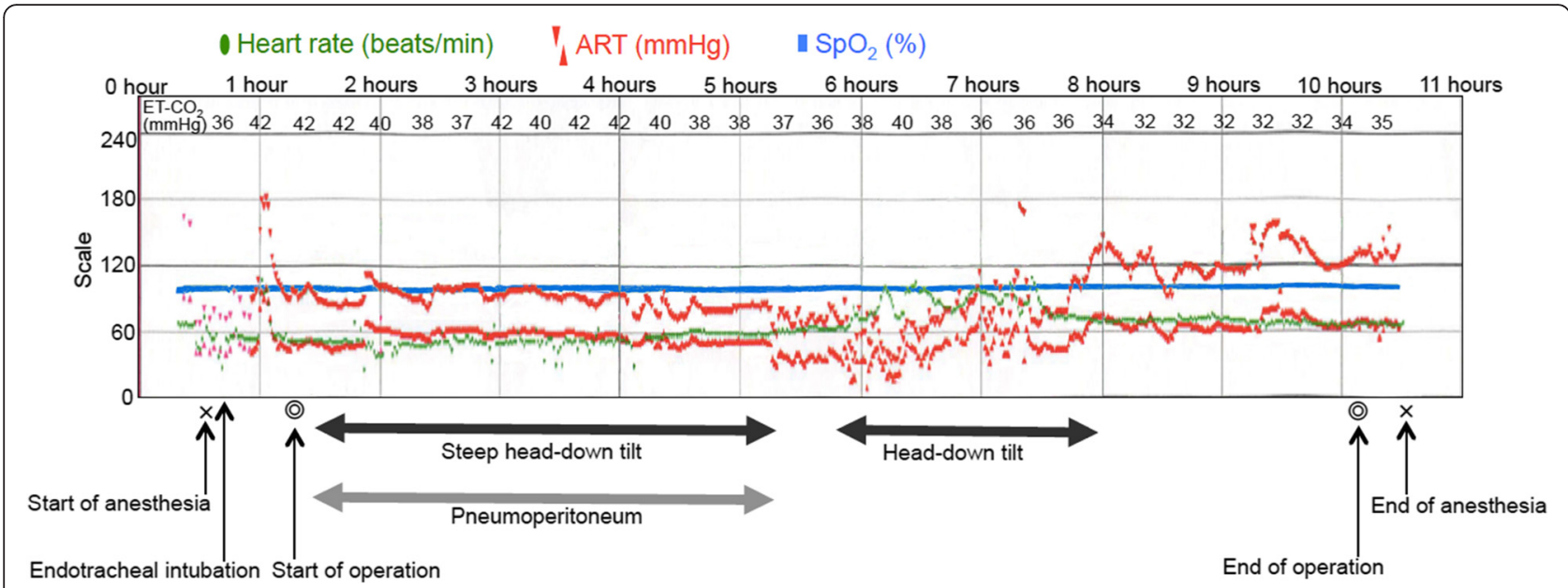

Fig. 1 Anesthetic chart. ART arterial blood pressure, $E T-\mathrm{CO}_{2}$ end-tidal carbon dioxide, $\mathrm{SpO}_{2}$ percutaneous oxygen saturation

was a rare occurrence of a hemorrhage resulting from an injury to a branch of the external iliac artery along the internal oblique muscle. We suspect that the vessel was transected during insertion of the laparoscope port at the beginning of surgery. The main reason the surgeon was unaware of the hemorrhage was the steep head-down tilt of the surgical table. We suspect that blood began to pool in our patient's superior abdomen during surgery, and that the hemorrhage was suppressed by the increased intra-abdominal pressure. When our patient was returned to the horizontal position and gas insufflation was ceased, the resulting increased rate of hemorrhage caused a sudden drop in blood pressure. There was the possibility that an air embolism caused sudden hypotension when the table returned to its horizontal position, because the surgical site was at a higher level than the heart. However, our patient's end-tidal carbon dioxide level did not change dynamically after the table was leveled. Anesthesiologists might have been able to recognize the gradual decrease in blood pressure caused by the hemorrhage. Although hemodynamics during RALP are complex, including increased venous return caused by the steep head-down tilt and restriction of this increase in venous return owing to pneumoperitoneum, two factors prevented us from recognizing the presence of a hemorrhage. First, the steep head-down tilt concealed the hemorrhage by increasing our patient's blood pressure. Second, unrestricted fluid management concealed the hypovolemia caused by bleeding. Restrictive fluid management is generally recommended to prevent complications, such as pulmonary edema, laryngeal edema, and central nervous system dysfunction $[9,10]$. However, restrictive fluid management is not performed in general in our institute at the surgeons' request.

Anesthesiologists should be aware that approximately one-third of complications in laparoscopic surgery occur between the time of camera insertion and insertion of the ports [11]. The most frequent complication, occurring in 0.5 cases out of 1000 , is injury to the retroperitoneal great vessels, including the abdominal aorta, inferior vena cava, common iliac artery, external iliac artery, and internal iliac artery. The second most frequent complication is intestinal injury, which occurs in 0.4 cases out of 1000. Injury to the abdominal wall blood vessels occurs less often $[11,12]$. Additionally, several cases of bleeding from the port site have been reported $[13,14]$. The abdominal wall blood vessels include the superficial epigastric artery and vein, superficial circumflex artery and vein, inferior epigastric artery, and deep circumflex iliac artery. Injuries to epigastric vessels, usually located between 4 and $8 \mathrm{~cm}$ from the midline and to vessels on the left side closer to the midline, have been reported previously [12]. Therefore, this area should be avoided as the entry point to the anterior abdominal wall. In most cases of injured epigastric vessels, bleeding at the port site is found as extraperitoneal hematoma or bleeding, and blood flows into the peritoneum through the defect created by the port when the port is removed. However, a hemorrhage in the present case was not found when the surgeon checked at the time of port removal. The hemorrhage was suspected only after the patient was returned to the horizontal position. Vital signs must be carefully monitored during and after alteration of patient position.

\section{Conclusions}

In this case, a hemorrhage was caused by injury to a branch of the abdominal wall vessels during RALP. The increased intra-abdominal pressure resulting from pneumoperitoneum, the normal blood pressure resulting from a steep head-down tilt, and unrestricted fluid management prevented prompt recognition of the hemorrhage. 


\section{Acknowledgements}

None.

\section{Funding}

Self-funding.

\section{Availability of data and materials}

Not applicable. There are no datasets used in this case report.

\section{Authors' contributions}

SN collected the data and wrote the case report. JN helped write the manuscript and edit the figure. TS and NK helped write the manuscript and assisted with the literature review. TM revised and approved the manuscript. All authors read and approved the final manuscript.

\section{Competing interests}

The authors declare that they have no competing interests.

\section{Consent for publication}

Written informed consent was obtained from the patient for publication of this case report and any accompanying images. A copy of the written consent is available for review by the Editor-in-Chief of this journal.

\section{Ethics approval and consent to participate}

The Ethics Committee of Osaka Medical College does not need ethics approval for one-case reports.

Received: 31 March 2016 Accepted: 9 August 2016

\section{Published online: 30 August 2016}

\section{References}

1. Parsons JK, Bennett JL. Outcomes of retropubic, laparoscopic, and roboticassisted prostatectomy. Urology. 2008;72:412-6.

2. Hong JY, Oh YJ, Rha KH, Park WS, Kim YS, Kil HK. Pulmonary edema after da Vinci-assisted laparoscopic radical prostatectomy: a case report. J Clin Anesth. 2010;22:370-2.

3. Saito J, Noguchi S, Matsumoto A, Jinushi K, Kasai T, Kudo T, et al. Impact of robot-assisted laparoscopic prostatectomy on the management of general anesthesia: efficacy of blood withdrawal during a steep Trendelenburg position. J Anesth. 2015:29:487-91.

4. Chalmers D, Cusano A, Haddock P, Staff I, Wagner J. Are preexisting retinal and central nervous system-related comorbidities risk factors for complications following robotic-assisted laparoscopic prostatectomy? Int Braz J Urol. 2015:41:661-8.

5. Weber ED, Colyer MH, Lesser RL, Subramanian PS. Posterior ischemic optic neuropathy after minimally invasive prostatectomy. J Neuroophthalmol. 2007;27:285-7

6. Djavan B, Agalliu I, Laze J, Sadri H, Kazzazi A, Lepor H. Blood loss during radical prostatectomy: impact on clinical, oncological and functional outcomes and complication rates. BJU Int. 2012;110:69-75.

7. Joo EY, Moon YJ, Yoon SH, Chin JH, Hwang JH, Kim YK. Comparison of acute kidney injury after robot-assisted laparoscopic radical prostatectomy versus retropubic radical prostatectomy: a propensity score matching analysis. Medicine (Baltimore). 2016;95:e2650.

8. Alemozaffar M, Sanda M, Yecies D, Mucci LA, Stampfer MJ, Kenfield SA. Benchmarks for operative outcomes of robotic and open radical prostatectomy: results from the Health Professionals Follow-up Study. Eur Urol. 2015;67:4328

9. Gainsburg DM. Anesthetic concerns for robotic-assisted laparoscopic radical prostatectomy. Minerva Anestesiol. 2012;78:596-604.

10. Awad H, Walker CM, Shaikh M, Dimitrova GT, Abaza R, O'Hara J. Anesthetic considerations for robotic prostatectomy: a review of the literature. J Clin Anesth. 2012;24:494-504

11. Deffieux X, Ballester M, Collinet $P$, Fauconnier A, Pierre F. French National College of Gynaecologists and Obstetricians. Risks associated with laparoscopic entry: guidelines for clinical practice from the French College of Gynaecologists and Obstetricians. Eur J Obstet Gynecol Reprod Biol. 2011:158:159-66.

12. Saber AA, Meslemani AM, Davis R, Pimentel R. Safety zones for anterior abdominal wall entry during laparoscopy: a CT scan mapping of epigastric vessels. Ann Surg. 2004;239:182-5
13. Ishikawa H, Yamaji S, Suzuki Y, Usui H, Mitsuhashi A, Shozu M. Late onset hematoma at the port site by a $5-\mathrm{mm}$ VersaStep ${ }^{\mathrm{TM}}$ bladeless trocar: a case report. Jpn J Gynecol Obstet Endosc. 2012;28:358-62. Japanese.

14. Hurd WW, Pearl ML, DeLancey JO, Quint EH, Garnett B, Bude RO. Laparoscopic injury of abdominal wall blood vessels: a report of three cases. Obstet Gynecol. 1993;82:673-6.

\section{Submit your next manuscript to BioMed Central and we will help you at every step:}

- We accept pre-submission inquiries

- Our selector tool helps you to find the most relevant journal

- We provide round the clock customer support

- Convenient online submission

- Thorough peer review

- Inclusion in PubMed and all major indexing services

- Maximum visibility for your research

Submit your manuscript at www.biomedcentral.com/submit 